\title{
Beauty of turbulent convection: A particle tracking endeavor
}

\author{
Philipp Godbersen $\odot,{ }^{*}$ Johannes Bosbach $\odot$, Daniel Schanz $\odot$, and Andreas Schröder \\ Department of Experimental Methods, Institute of Aerodynamics and Flow Technology, \\ German Aerospace Center (DLR), 37073 Göttingen, Germany
}

(Received 13 August 2021; published 15 November 2021)

\begin{abstract}
This paper is associated with a video winner of a 2020 American Physical Society's Division of Fluid Dynamics (DFD) Gallery of Fluid Motion Award for work presented at the DFD Gallery of Fluid Motion. The original video is available online at the Gallery of Fluid Motion, https://doi.org/10.1103/APS.DFD.2020.GFM.V0074.
\end{abstract}

DOI: 10.1103/PhysRevFluids.6.110509

Rayleigh-Bénard convection (RBC), the flow in a layer of fluid heated from below and cooled from above, is a prevalent model system to study the fundamentals of thermal convection [1]. Characteristic of the turbulent RBC system is the occurrence of a large-scale circulation (LSC), which develops by self-organization of the thermal plumes erupting from the thermal boundary layers. In cylindrical samples of aspect ratios close to one, the LSC reveals complex short- and long-term dynamics involving rotations, cessations, and restarts [2], torsional oscillations [3,4], and sloshing modes [4]. While these have been studied intensively employing local temperature probes in the past, volumetric measurements of the LSC, allowing for direct insight into the underlying turbulent processes, are still rare [5].

To bridge this gap, we perform Lagrangian particle tracking (LPT) in a dedicated convection experiment using air as the working fluid by combining an in-line multicamera setup, long-lasting helium-filled soap bubbles, and high-power pulsed light-emitting diode arrays [6]. The convection cell with a sample height of $1.1 \mathrm{~m}$ has an aspect ratio of one and hence spans a volume of $1 \mathrm{~m}^{3}$. It allows us to generate classical turbulent RBC and to perform time-resolved LPT in the complete sample volume (Fig. 1). At moderate temperature differences up to $12 \mathrm{~K}$, we study $\mathrm{RBC}$ at Rayleigh numbers up to $\mathrm{Ra}=1.5 \times 10^{9}(\mathrm{Pr}=0.70)$. By using the Shake-The-Box Lagrangian particle tracking algorithm [7], we could track instantaneously up to 560000 particles covering the complete sample. With the FlowFit data assimilation scheme [8] we interpolate the scattered velocity and acceleration data on cubic grids, using physical regularizations to further increase the spatial resolutions. The resulting data sets allow for Lagrangian and Eulerian statistics of turbulent quantities as well as visualizations of the LSC and smaller-scale turbulent structures, such as thermal plumes and turbulent background fluctuations. Within our video we begin by showing the scattered velocity data of the tracked particles color coding the vertical velocity in order to give an overview of the turbulent flow with its dominating LSC. Visualizing particle tracks in the form of trails provides a more detailed view into the flow making complex flow behavior within the LSC, as well as the characteristic corner rollers, readily apparent. Additional insight into the turbulent transport and mixing processes can be gained from the isosurfaces of the $Q$ criterion, made accessible by the

\footnotetext{
*Philipp.Godbersen@dlr.de
}

Published by the American Physical Society under the terms of the Creative Commons Attribution 4.0 International license. Further distribution of this work must maintain attribution to the author(s) and the published article's title, journal citation, and DOI. 


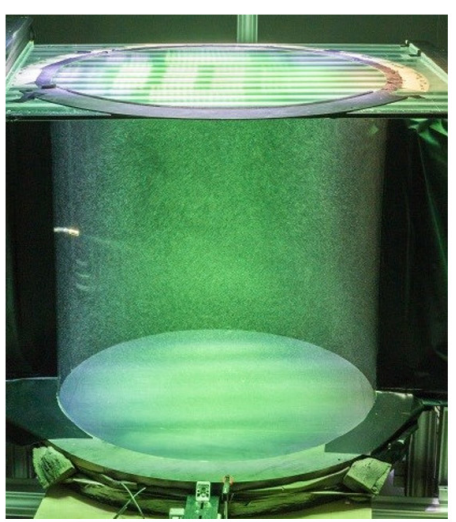

(a)

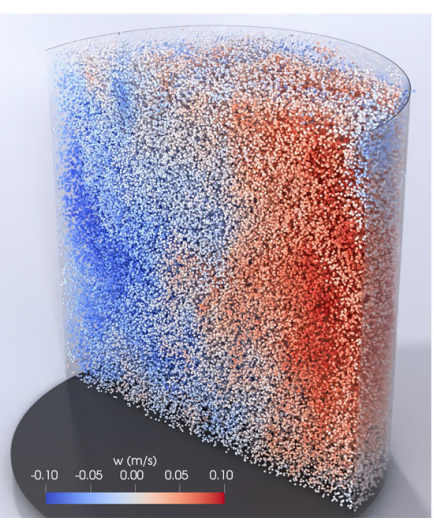

(b)

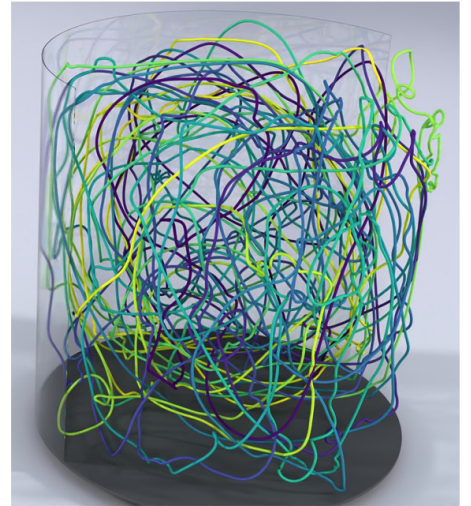

(c)

FIG. 1. (a) Photo of the RBC cell with long-lived, microscopic helium-filled soap bubbles. (b) Tracked particles in the half volume colored by vertical velocity at $\mathrm{Ra}=5.3 \times 10^{8}$. (c) Visualization of a single long track throughout the measurement volume over 44000 images $\left(\mathrm{Ra}=1.5 \times 10^{9}\right.$; color shows progression in time).

FlowFit processing. This visualization of the vortical structures also has the benefit that the space within the volume is visible more clearly as the very dense particle visualizations shown earlier tend to occlude this. As the RBC cell is captured in its entirety at high spatial and temporal resolution, we are able to study the complex interplay of the dynamics between the LSC and smaller turbulent structures. In the visualization of the entire fluid sample we can identify vortical structures generated above the heated bottom plate, which then get swept away by the dominating motion of the LSC [Fig. 2(b)].

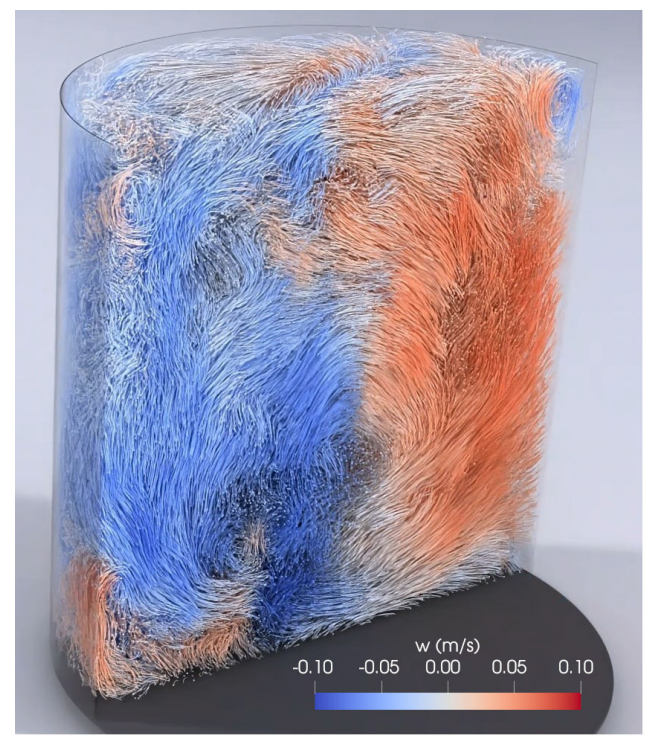

(a)

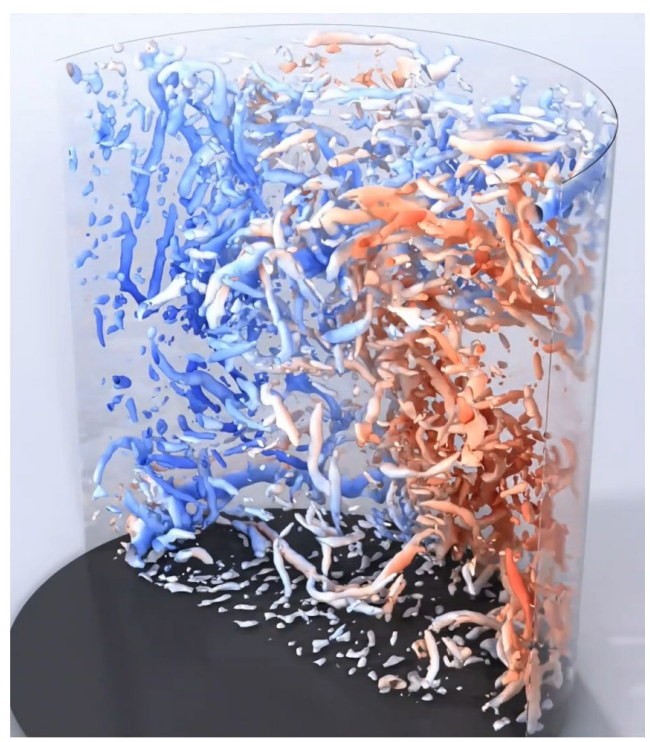

(b)

FIG. 2. Lagrangian and Eulerian visualizations of turbulent structures, displaying one half of the cylinder $\left(\mathrm{Ra}=1.5 \times 10^{9}\right)$. (a) Particles with trails to indicate turbulent structures on small and intermediate scales within the dominating LSC. (b) Isosurfaces of $Q$ criterion revealing vortical substructures inside the LSC. 


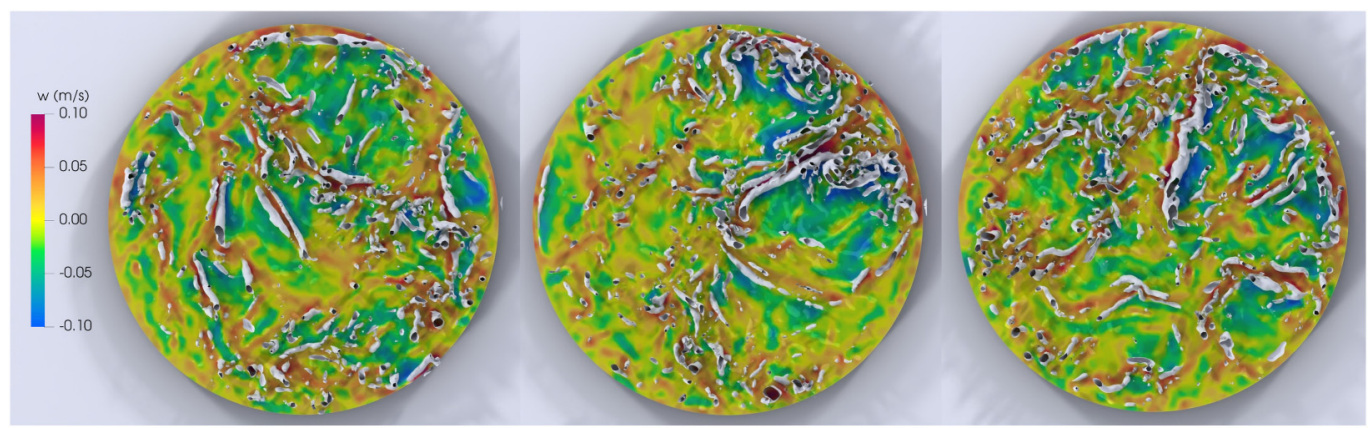

FIG. 3. Vertical velocity field visualized in a slice above the bottom plate together with isosurfaces of the $Q$ criterion revealing the fingerprints of thermal line plumes rising from the thermal boundary layer and inducing pairs of elongated vortices $\left(\mathrm{Ra}=1.5 \times 10^{9}\right)$.

Using the same data set, we then focus on a detailed view of the region above the bottom plate (Fig. 3) to further investigate these turbulent interactions. First to note is the presence of the footprints of the thermal plumes as linear structures in the fields of the vertical velocity component. The rising line plumes generate pairwise elongated vortical structures which are initiated by instabilities in the adjacent shear layers and fed by the air flow entrained from around the rising plume. Further analysis of the creation and dynamic behavior of these structures is the subject of ongoing studies. Herewith a multitude of prominent flow features are present in this region, consisting of, besides the aforementioned plumes, dust devils and corner rollers, all dynamically interacting with the LSC. The visualizations shown here provide a glimpse into the wealth of the data collected, giving ample opportunity for further, more systematic investigations using this data set.

Support with hardware and software for illumination and image acquisition by LaVision GmbH is gratefully acknowledged. We thank J. Lemarechal, T. Kleindienst, and C. Fuchs for their contributions to the RBC sample and J. Agocs for his support during setup of the optical system. J.B. acknowledges several constructive conversations with S. Weiss. We thank the Max-PlanckInstitute DS for provision of the refrigerated bath circulator. This work was supported by the Deutsche Forschungsgemeinschaft (DFG) through Grant No. SCHR 1165/5-1 as part of the Priority Programme on Turbulent Superstructures (DFG SPP 1881).

[1] F. Chillà and J. Schumacher, New perspectives in turbulent Rayleigh-Bénard convection, Eur. Phys. J. E 35, 1 (2012).

[2] E. Brown and G. Ahlers, Rotations and cessations of the large-scale circulation in turbulent RayleighBénard convection, J. Fluid Mech. 568, 351 (2006).

[3] D. Funfschilling, E. Brown, and G. Ahlers, Torsional oscillations of the large-scale circulation in turbulent Rayleigh-Bénard convection, J. Fluid Mech. 607, 119 (2008).

[4] Q. Zhou, H.-D. Xi, S.-Q. Zhou, C. Sun, and K.-Q. Xia, Oscillations of the large-scale circulation in turbulent Rayleigh-Bénard convection: The sloshing mode and its relationship with the torsional mode, J. Fluid Mech. 630, 367 (2009).

[5] G. Paolillo, C. S. Greco, T. Astarita, and G. Cardone, Experimental determination of the 3-D characteristic modes of turbulent Rayleigh-Bénard convection in a cylinder, J. Fluid Mech. 922, A35 (2021).

[6] J. Bosbach, D. Schanz, P. Godbersen, and A. Schröder, in Proceedings of the 14th International Symposium on Particle Image Velocimetry, Chicago, 2021 (Illinois Tech, 2021), Vol. 1. 
[7] D. Schanz, S. Gesemann, and A. Schröder, Shake-the-box: Lagrangian particle tracking at high particle image densities, Exp. Fluids 57, 70 (2016).

[8] S. Gesemann, F. Huhn, D. Schanz, and A. Schröder, Proceedings of the 18th International Symposium on Applications of Laser and Imaging Techniques to Fluid Mechanics, Lisbon, 2016 (unpublished), pp. 4-7. 